\title{
Analysis of Circulating Apoptosis Mediators and Proinflammatory Cytokines in Patients With Idiopathic Hypertrophic Cardiomyopathy
}

\section{Comparison Between Nonobstructive and Dilated-Phase Hypertrophic Cardiomyopathy}

\author{
Kan ZEN, ${ }^{1} \mathrm{MD}$, Hidekazu IRIE, ${ }^{1} \mathrm{MD}$, Tomoki DouE, ${ }^{1} \mathrm{MD}$, \\ Michitaka TAKAmIYA, ${ }^{1} \mathrm{MD}$, Tetsuhiro YAMANO, ${ }^{1} \mathrm{MD}$, Takahisa SAWADA, ${ }^{1} \mathrm{MD}$, \\ Akihiro AZUMA, ${ }^{1} \mathrm{MD}$, and Hiroaki MATSUBARA, ${ }^{1} \mathrm{MD}$
}

\section{SUMMARY}

We examined the plasma levels of soluble Fas (sFas) or Fas ligand (sFas-L), tumor necrosis factor-alpha (TNF- $\alpha$ ), and interleukin-6 (IL-6) in patients with idiopathic nonobstructive (HNCM) and dilated-phase (DHCM) hypertrophic cardiomyopathy.

Patients with idiopathic hypertrophic cardiomyopathy (HCM) may deteriorate to DHCM and the pathogenesis is unknown.

The levels of these plasma cytokines were measured by ELISA and echocardiography was performed in $38 \mathrm{HNCM}$ and 11 DHCM patients, and 10 normal subjects. The followup period was three years.

In HNCM, TNF- $\alpha(43.3 \pm 45.2$ versus $16.9 \pm 4.3 \mathrm{pg} / \mathrm{mL})$ and IL-6 (65.1 \pm 86.4 versus $4.0 \pm 2.1 \mathrm{pg} / \mathrm{mL})$ were slightly higher compared to normal subjects and sFas $(3.7 \pm 1.2$ versus $2.1 \pm 0.7 \mathrm{ng} / \mathrm{mL}$ ) increased significantly. sFas (3.9 \pm 1.8$)$, TNF- $\alpha(79.3 \pm 72.4)$, and IL-6 (234.1 \pm 135.2$)$ in DHCM were significantly increased and only IL-6 was significantly different from HNCM. sFas-L $(0.18 \pm 0.08$ versus $0.25 \pm 0.05 \mathrm{ng} / \mathrm{mL})$ in HNCM was significantly decreased, and the decrease was marked in DHCM $(0.05 \pm$ 0.02). In HNCM, TNF- $\alpha$ was negatively correlated with fractional shortening $(\mathrm{r}=-0.432$, $P=0.0062)$ or positively with IL-6 $(\mathrm{r}=0.665, P<0.0001)$, while sFas-L was negatively correlated with IL-6 ( $\mathrm{r}=-0.580, P<0.0001)$. DHCM with high sFas had significantly higher cumulative incidences of worsening heart failure.

The Fas/Fas-L system and proinflammatory cytokines may play an important role in the status of HCM and its progression to DHCM. (Int Heart J 2005; 46: 231-244)

Key words: Dilated-phase hypertrophic cardiomyopathy, Soluble Fas, Soluble Fas ligand, Tumor necrosis factor-alpha, Interleukin-6

From the ${ }^{1}$ Department of Cardiovascular Medicine, Kyoto Prefectural University of Medicine, Kyoto, Japan.

Address for correspondence: Kan Zen, MD, Department of Cardiovascular Medicine, Kyoto Prefectural University of Medicine, Kajii-cho 465, Kawaramachi Hirokoji Agaru, Kamigyou-ku, Kyoto 602-8566, Japan.

Received for publication October 27, 2004.

Revised and accepted November 8, 2004. 
IDIOPATHIC hypertrophic cardiomyopathy (HCM) is a primary cardiac disorder, often transmitted genetically, with heterogeneous clinical and morphological expression, and most patients have good prognoses. However, systolic ventricular dysfunction and congestive heart failure (CHF), usually associated with left ventricular remodeling demonstrated as wall thinning and chamber enlargement, occur in about 5 percent of the patients, and the condition was previously known as dilated-phase hypertrophic cardiomyopathy (DHCM) ${ }^{1,2)}$ DHCM is a refractory disease and the prognosis is extremely poor compared to other types of HCM. ${ }^{2}$ Although many factors are thought to be related to the pathogenesis of DHCM, ${ }^{3-5)}$ the details are still unknown. Persistent increments in circulating levels of some cardiac enzymes are often observed in DHCM patients and they show abnormal thallium perfusion or antimyosin uptake in myocardial scintigraphy. ${ }^{6,7)}$ Furthermore, pathological studies have revealed that cardiac myocyte degeneration with marked fibrosis was observed in a broad area of ventricular myocardium ${ }^{3)}$ and immunohistochemical evaluation of apoptosis showed apoptotic cells and bodies in the destroyed myocytes along the border between the fibrotic area and myofibrils in DHCM patients. ${ }^{8)}$ These findings suggest the presence of ongoing apoptotic changes in cardiac myocytes of DHCM.

Recent studies have reported the overexpression of soluble apoptosis mediators and proinflammatory cytokines in patients with several cardiac diseases. ${ }^{9-14)}$ Circulating apoptosis mediators, such as soluble Fas (sFas), an inhibitor of apoptosis, ${ }^{15)}$ and soluble Fas ligand (sFas-L), an inducer of apoptosis, ${ }^{16)}$ were found to be elevated in CHF and well correlated with the severity of symptoms and prognosis of the patients. ${ }^{9,11,17)}$ Proinflammatory cytokines, such as tumor necrosis factor-alpha (TNF- $\alpha$ ) and interleukin-6 (IL-6), are capable of modulating cardiac function by a variety of mechanisms including induction of cardiac myocyte apoptosis. ${ }^{13,18)}$ In fact, Narula, et al reported that progressive loss of cardiac myocytes due to apoptosis would contribute to the deterioration of myocardial function in CHF. ${ }^{19)}$ Thus, apoptosis plays an important role in a variety of cardiovascular diseases. ${ }^{20)}$

We hypothesized that progressive cardiac myocyte loss due to apoptosis and detrimental effects by proinflammatory cytokines in the myocardium might be important factors in the status and pathogenesis of DHCM. In the present study, we sought to examine the plasma levels of these apoptosis mediators and proinflammatory cytokines in HCM patients, including DHCM, and investigated their relations to each other and to other clinical variables, such as cardiac natriuretic peptides, soluble interleukin- 2 receptor (sIL-2R) as a marker of T-cell activation, and echocardiographic data to validate the hypothesis. 


\section{Methods}

Study subjects: We prospectively studied 38 patients with hypertrophic nonobstructive cardiomyopathy (HNCM) and eleven DHCM patients for three years. Ten age- and gender-matched normal subjects without diseases and medications were also included. All patients were receiving regular outpatient treatment in our hospital (Kyoto Prefectural University of Medicine Hospital) and their clinical courses were stable for six months at least. They were diagnosed as HCM after studying their history of illness, physical examination, echocardiography, and previously performed cardiac catheterization. In the case where the left ventricular diastolic dimension of the HCM patient increased to 55 millimeters or more, the left ventricular ejection fraction decreased to 50 percent or less using echocardiography during his clinical course and the patient was confirmed not to have any coronary artery disease. We diagnosed the patient as having DHCM in our hospital. Patients with significant concomitant disease, such as pulmonary disease, malignancy, autoimmune disorders, neurodegenerative disorders, diabetes mellitus, thyroid disease, or concurrent viral disease were excluded. All serum creatinine and aminotransferase levels were within the normal ranges. All subjects studied provided informed consent to the study, which was approved by the Ethics Review Committee on Human Research of Kyoto Prefectural University of Medicine.

Blood sampling and measurement of plasma sFas, sFas-L, TNF- $\alpha$, and IL-6 levels: After an overnight fast, peripheral venous blood samples were obtained from the antecubital vein and transferred to chilled disposable tubes containing 2-sodiumethylenediamine tetraacetic acid $(1.5 \mathrm{mg} / \mathrm{mL})$. The tubes were promptly centrifuged at $4{ }^{\circ} \mathrm{C}$, and aliquots of plasma were immediately stored at $-80^{\circ} \mathrm{C}$ until analyzed. Plasma sFas and sFas-L levels were measured with commercially available enzyme-linked immunosorbent assay (ELISA) kits (Medical \& Biological Laboratories Co., Ltd, Nagoya, Japan). The sFas and sFas-L ELISA kits were based on a sandwich ELISA and could specifically detect sFas and sFas-L. Plasma TNF- $\alpha$ and IL-6 levels were also measured using sandwich ELISA kits with two monoclonal anti-human TNF- $\alpha$ and IL-6 antibodies (Immunotech, Marseille, France). The sensitivity limit of the assay was $500 \mathrm{pg} / \mathrm{mL}$ for sFas, $30 \mathrm{pg} / \mathrm{mL}$ for sFas-L, $5 \mathrm{pg} / \mathrm{mL}$ for TNF- $\alpha$, and $3 \mathrm{pg} / \mathrm{mL}$ for IL- 6 according to the manufacturer. We performed all assays in duplicate, and the mean of two sets of data was determined for each individual sample. Using the ELISA method with commercial kits, the intra - and interassay coefficients of variation $(\mathrm{CV})$ were $1.49-7.12 \%$ and 3.81 8.14\% for sFas, $4.2-7.4 \%$ and $6.3-10.7 \%$ for sFas-L, $1.6-10 \%$ and $5.4-12.8 \%$ for TNF- $\alpha$ and $1.6-6.8 \%$ and $7.9-14.6 \%$ for IL-6.

Measurement of plasma cardiac natriuretic peptides and sIL-2R levels: Plasma atrial natriuretic peptide (ANP) and brain natriuretic peptide (BNP) levels were 
measured with a specific immunoradiometric assay for human ANP or BNP using commercial kits (Shionoria kit, Shionogi and Co., Ltd., Japan). Normal values were less than $43 \mathrm{pg} / \mathrm{mL}$ for ANP and $18.4 \mathrm{pg} / \mathrm{mL}$ for BNP when using these assay kits. The intra- and interassay coefficients of variation $(\mathrm{CV})$ were $5.1 \%$ and $5.8 \%$ for ANP, and 5.2\% and 6.1\% for BNP. Plasma sIL-2R levels were also measured using a sandwich ELISA kit with two monoclonal anti-human IL-2R antibodies (IMMULITE IL-2R diagnostic test kit, Diagnostic Products Corporation United Kingdom). Normal values were within $220-530 \mathrm{U} / \mathrm{mL}$ using this assay kit, and the intra- and interassay coefficients of variation $(\mathrm{CV})$ were $4.5-8.0 \%$ and $3.3-6.4 \%$.

Echocardiographic study: Two-dimensional, M-mode, and Doppler echocardiographic studies were performed by standard methods at the time of blood sampling, and conventional parasternal and apical views were obtained. Patients were imaged with a Hewlett-Packard ultrasound system equipped with a multifrequency transducer (2.5 and 3.5 MHz). Some parameters were measured with $\mathrm{M}$ mode and Doppler echocardiography, such as interventricular septal thickness (IVSTd), left ventricular end-diastolic dimension (LVDd), fractional shortening (FS), left atrial dimension (LAD), ratio of early to late diastolic transmitral flow (E/A), and mitral E wave deceleration time (DT) by standard methods.

Statistical analysis: The data classified according to HCM subclasses and normal subjects were analyzed. End points for follow-up were hospitalization for deterioration of $\mathrm{CHF}$, cardiac death, or cardiac sudden death. All values are expressed as the mean $\pm \mathrm{SD}$. Categorical data were compared using one-way analysis of variance (ANOVA) for repeated measures followed by Scheffe's procedure for post hoc comparison. The Mann-Whitney U test was used for paired data. A simple linear regression analysis was performed to determine the relationship between continuous variables by the least-squares method. Kaplan-Meier analysis was performed on the cumulative incidence of CHF in DHCM patients stratified into two groups on the basis of the median values of plasma blood sampling levels and echocardiographic data and differences between the cumulative incidence curves were analyzed using the Breslow-Gehan-Wilcoxon test. A $P$ value $<0.05$ was accepted as statistically significant.

\section{RESULTS}

Patient characteristics, echocardiographic data, and plasma levels of cardiac natriuretic peptides: Table I shows the clinical characteristics, echocardiographic data, and plasma levels of cardiac natriuretic peptides for both HCM patient groups and the normal subjects. There were no significant differences in age or gender among the three groups and most patients had received $\beta$-blockers or ver- 
Table I. Clinical Characteristics, Echocardiographic Parameters, and Plasma Levels of Cardiac Natriuretic Peptides for Both Patient Groups with Hypertrophic Cardiomyopathy and Normal Subjects

\begin{tabular}{lccc}
\hline Variable & Normal subjects $(n=10)$ & HNCM $(n=38)$ & DHCM $(n=11)$ \\
\hline Age & $56 \pm 6$ & $61 \pm 13$ & $57 \pm 10$ \\
Sex (male / female) & $7 / 3$ & $27 / 11$ & $9 / 2$ \\
NYHA (I / II / III / IV) & - & $36 / 2 / 0 / 0$ & $0 / 9 / 2 / 0$ \\
Echocardiography & & & \\
$\quad$ IVSTd (mm) & $9.2 \pm 0.8$ & $17.2 \pm 4.5^{\#}$ & $15.1 \pm 5.1^{\#}$ \\
$\quad$ LVDd (mm) & $48.2 \pm 2.7$ & $46.7 \pm 4.7$ & $59.2 \pm 5.0^{\# \S}$ \\
FS (\%) & $38.2 \pm 4.4$ & $38.9 \pm 7.0$ & $18.0 \pm 3.8^{\# \S}$ \\
LAD (mm) & $37.2 \pm 2.2$ & $43.0 \pm 6.1^{\#}$ & $49.5 \pm 6.2^{\# \S}$ \\
$\quad$ E/A & $1.04 \pm 0.24$ & $0.96 \pm 0.41$ & $1.97 \pm 1.69^{\# \S}$ \\
$\quad$ DT (msec) & $209.2 \pm 26.3$ & $232.0 \pm 84.1$ & $225.9 \pm 136.2$ \\
Cardiac natriuretic peptides & & & \\
$\quad$ ANP levels (pg/mL) & $20.4 \pm 8.9$ & $62.4 \pm 44.5$ & $110.6 \pm 77.6^{\# \S}$ \\
$\quad$ BNP levels (pg/mL) & $10.9 \pm 4.1$ & $211.6 \pm 187.3^{\#}$ & $384.8 \pm 264.7^{\# \S}$ \\
Cardioactive drugs & - & $22(58)$ & $9(82)$ \\
$\quad \beta$-Blocker & - & $12(32)$ & $4(36)$ \\
$\quad$ Verapamil & - & 0 & $2(18)$ \\
$\quad$ Diuretics & & &
\end{tabular}

Values are mean $\pm \mathrm{SD}$ or $n(\%)$. \#: $P<0.05$ versus normal subjects. $\S: P<0.05$ versus HNCM group. $\mathrm{HNCM}=$ hypertrophic nonobstructive cardiomyopathy; DHCM = dilated-phase hypertrophic cardiomyopathy; NYHA = New York Heart Association; IVSTd = interventricular septal thickness; $\mathrm{LVDd}=$ left ventricular end-diastolic dimension; $F S=$ fractional shortening; $\mathrm{LAD}$ = left atrial dimension; $\mathrm{E} / \mathrm{A}=$ ratio of early to late diastolic transmitral flow; $\mathrm{DT}=$ mitral $\mathrm{E}$ wave deceleration time; $\mathrm{ANP}=$ atrial natriuretic peptide; $\mathrm{BNP}=$ brain natriuretic peptide.

apamil. Thirty-six of $38 \mathrm{HNCM}$ patients were classified as New York Heart Association (NYHA) functional class I and 2 as class II. Of the 11 DHCM patients, 9 were classified as NYHA functional class II and 2 as class III.

Echocardiographically, IVSTd in HNCM and DHCM patients was significantly increased compared to the normal subjects. LVDd, FS, LAD, and E/A in DHCM patients were significantly different from those in HNCM and normal subjects. LAD in HNCM patients was significantly increased compared to the normal subjects.

Plasma levels of ANP and BNP were significantly higher in DHCM patients than in HNCM and normal subjects. Plasma BNP levels in HNCM patients were significantly increased compared to normal subjects.

Plasma levels of apoptosis mediators, proinflammatory cytokines, and sIL-2R: Grouped values for the plasma levels of sFas, sFas-L, TNF- $\alpha$, IL-6, and sIL-2R are presented in Figure 1. Plasma sFas, TNF- $\alpha$, and IL-6 levels were significantly higher in DHCM patients (sFas: $3.87 \pm 1.77 \mathrm{ng} / \mathrm{mL}$ TNF- $\alpha$ : $79.3 \pm 72.4 \mathrm{pg} / \mathrm{mL}$ 

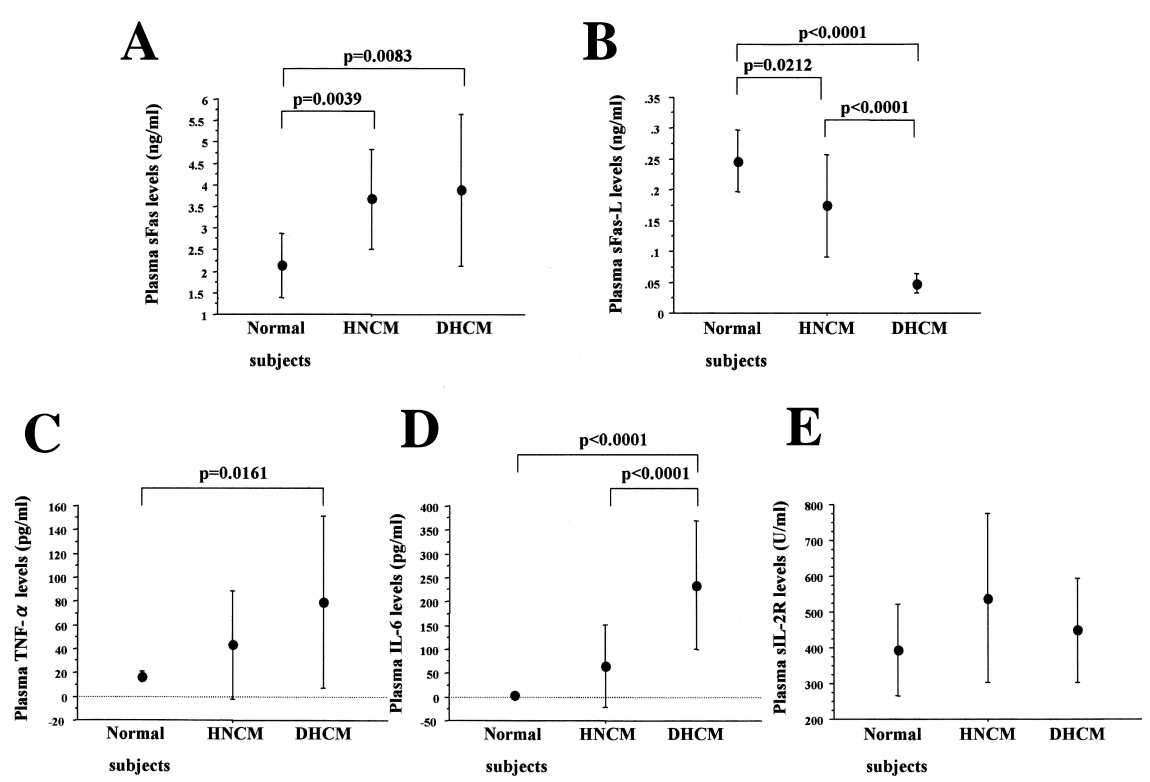

Figure 1. Plasma levels of apoptosis mediators, proinflammatory cytokines, and soluble interleukin-2 receptor (sIL-2R) in both groups of hypertrophic cardiomyopathy patients and normal subjects.

A: Plasma soluble Fas (sFas) levels increased significantly in patients with hypertrophic nonobstructive cardiomyopathy (HNCM) or dilated-phase hypertrophic cardiomyopathy (DHCM) compared to normal subjects and the differences between the HNCM and DHCM patient groups were not significant. B: Plasma soluble Fas ligand (sFas-L) levels decreased significantly in patients with HNCM or DHCM compared to normal subjects and the differences between the HNCM and DHCM patient groups were statistically significant. C: Plasma tumor necrosis factor-alpha (TNF- $\alpha$ ) levels increased significantly in DHCM patients compared to normal subjects and there were no significant differences between the patients with HNCM and DHCM and the normal subjects. D: Plasma interleukin-6 (IL-6) levels increased significantly in patients with DHCM compared to HNCM or normal subjects and there was no significant difference between the HNCM patients and normal subjects. E: There were no significant differences in plasma sIL-2R levels among the 3 groups.

IL-6: $234.1 \pm 135.2 \mathrm{pg} / \mathrm{mL}$ ) than in the normal subjects (sFas: $2.13 \pm 0.73 \mathrm{ng} / \mathrm{mL}$ TNF- $\alpha: 16.9 \pm 4.3 \mathrm{pg} / \mathrm{mL}$ IL-6: $4.0 \pm 2.1 \mathrm{pg} / \mathrm{mL}$ ). There were slightly higher levels of sFas, TNF- $\alpha$, and IL-6 in HNCM patients (sFas: $3.67 \pm 1.15 \mathrm{ng} / \mathrm{mL}$ TNF$\alpha: 43.3 \pm 45.2 \mathrm{pg} / \mathrm{mL}$ IL-6: $65.1 \pm 86.4 \mathrm{pg} / \mathrm{mL}$ ) compared to the normal subjects, but only the difference in sFas was statistically significant. Plasma sFas, TNF- $\alpha$, and IL-6 levels were slightly higher in DHCM than in HNCM patients but only the difference in IL-6 was statistically significant. The plasma levels of sFas-L were decreased significantly in $\operatorname{HNCM}(0.18 \pm 0.08 \mathrm{ng} / \mathrm{mL})$ and $\operatorname{DHCM}(0.05 \pm$ $0.02 \mathrm{ng} / \mathrm{mL})$ patients compared to normal subjects $(0.25 \pm 0.05 \mathrm{ng} / \mathrm{mL})$ and they were significantlyt lower in DHCM than in HNCM patients. Plasma sIL-2R levels were not significantly different among the 3 groups (HNCM: 538.0 $\pm 235.4 \mathrm{U} /$ mL DHCM: $449.5 \pm 145.8 \mathrm{U} / \mathrm{mL}$ normal subjects: $392.5 \pm 128.3 \mathrm{U} / \mathrm{mL})$. 


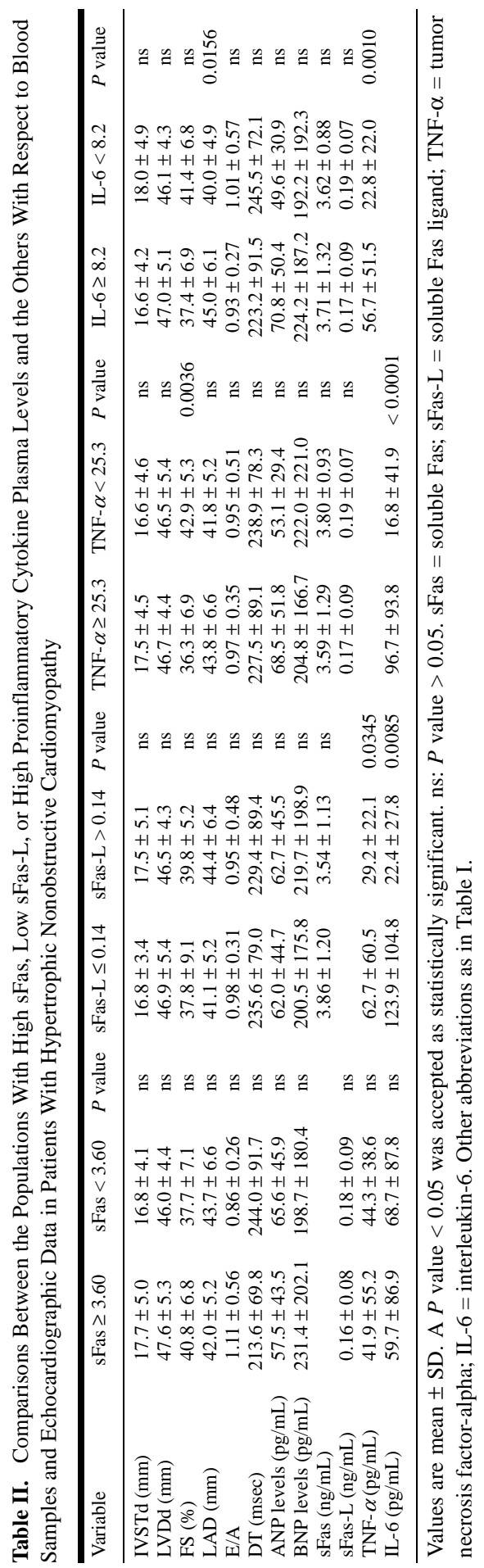


Comparisons between the populations with high sFas, low sFas-L, or high proinflammatory cytokine plasma levels and those without with respect to the blood samplings and echocardiographic data in HNCM patients are shown in Table II. Significant increases $(>+2 \mathrm{SD})$ in plasma sFas, TNF- $\alpha$, and IL-6 levels compared to normal subjects were seen in $39 \%, 61 \%$, and $61 \%$ for IL-6 of HNCM patients for sFas, TNF- $\alpha$, and IL-6, respectively. A significant decrease in plasma sFas-L levels of greater than $-2 \mathrm{SD}$ of that in normal subjects was seen in $42 \%$ of the HNCM patients. The low plasma sFas-L population (sFas-L $\leq 0.14$
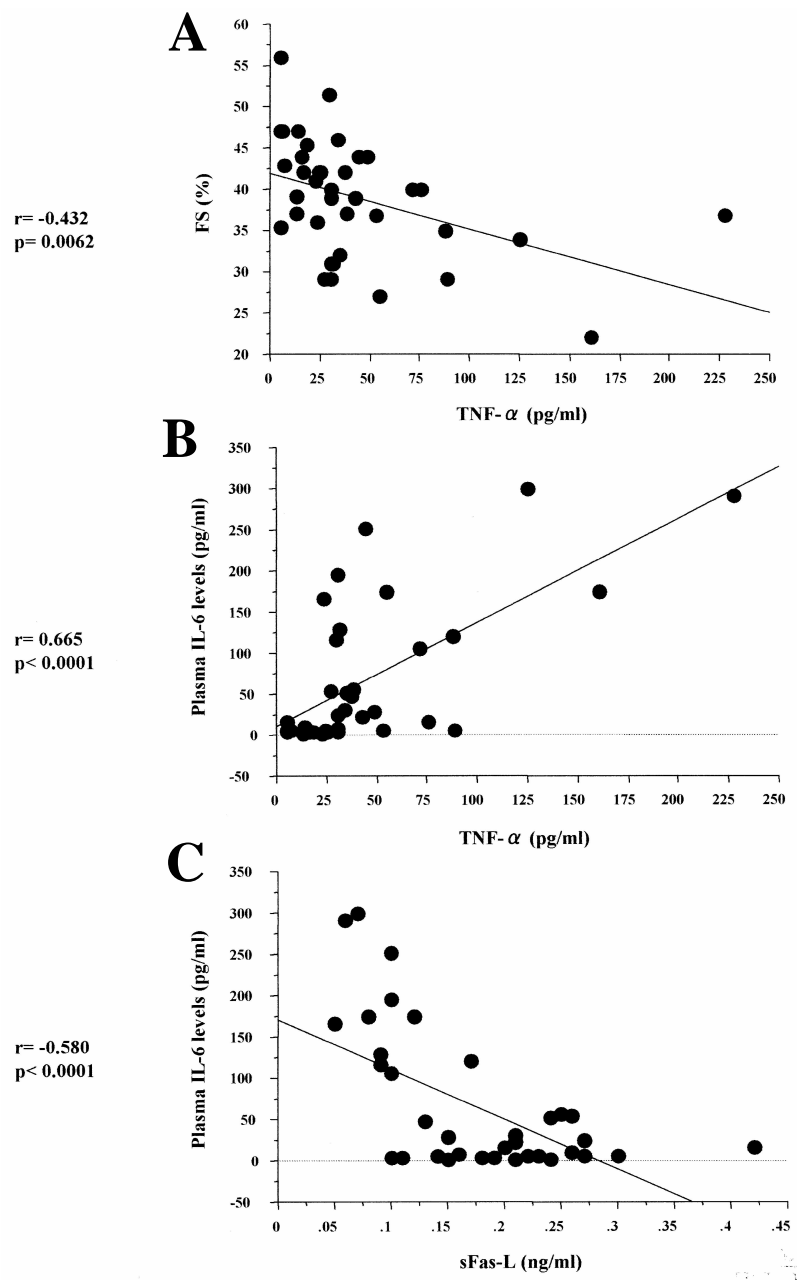

Figure 2. Correlation analysis among the blood samples and echocardiographic data in HNCM patients

(A) Plasma TNF- $\alpha$ levels negatively correlated with fractional shortening (FS) and (B) positively correlated with plasma IL-6 levels. (C) Plasma sFas-L levels were negatively correlated with plasma IL- 6 levels. These coefficients of correlation were statistically significant. Other abbreviations as in Figure 1. 


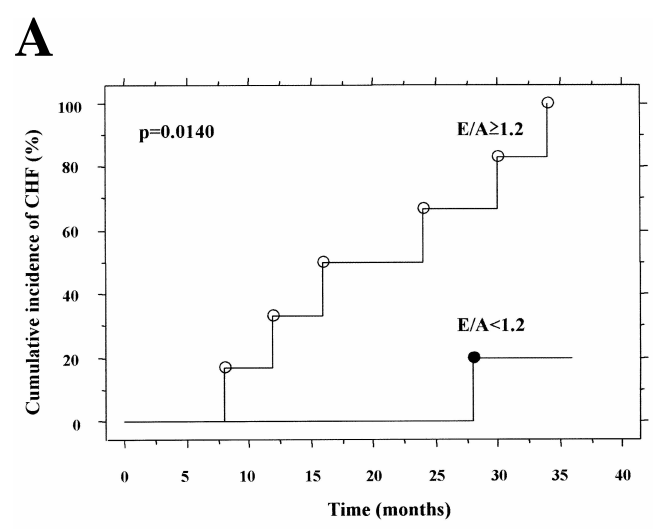

B

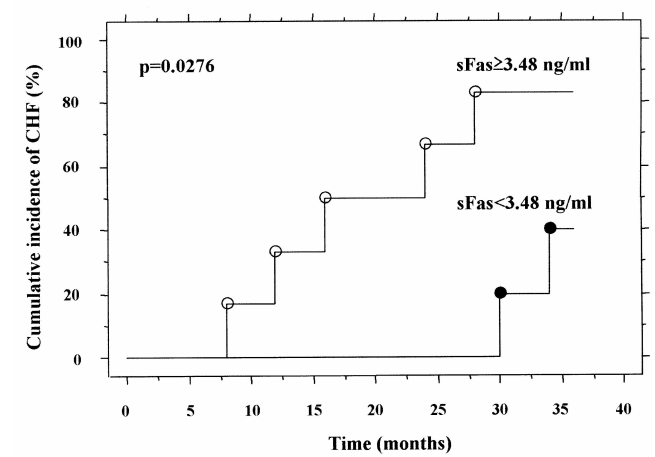

Figure 3. Kaplan-Meier plots showing the cumulative incidence of worsening congestive heart failure (CHF) in DHCM patients with a ratio of early to late diastolic transmitral flow (E/A) above and below 1.2 or plasma $\mathrm{sFas}$ levels above and below $3.6 \mathrm{ng} / \mathrm{mL}$. DHCM patients with high E/A (A) and sFas levels (B) had significantly higher cumulative incidences of worsening CHF than those of the other populations. Other abbreviations as in Figure 1.

$\mathrm{ng} / \mathrm{mL}$ ) of HNCM patients showed significant increases in plasma TNF- $\alpha$ and IL-6 levels compared to the other populations. There were significant decreases in FS and increases in plasma IL-6 levels in HNCM patients with high TNF- $\alpha$ levels (TNF- $\alpha \geq 25.3 \mathrm{pg} / \mathrm{mL}$ ) compared to those without. LAD and plasma TNF$\alpha$ levels were significantly higher in HNCM patients with high IL-6 levels (IL-6 $\geq 8.2 \mathrm{pg} / \mathrm{mL}$ ) than those without. In patients with DHCM, significant increases in plasma sFas and TNF- $\alpha$ were seen (45\% for sFas and $91 \%$ for TNF- $\alpha$ ). In addition, all DHCM patients showed significant increases in plasma IL-6 levels and decreases in sFas-L. A significant decrease in DT was observed in the high plasma sFas level population ( $\mathrm{sFas} \geq 3.60 \mathrm{ng} / \mathrm{mL}$ ) of DHCM patients compared to the other DHCM patients (data not shown). 
Correlation analysis among each blood sampling and echocardiographic data: In HNCM patients, plasma TNF- $\alpha$ levels were negatively correlated with FS (Figure 2A) and positively correlated with plasma IL-6 levels (Figure 2B). Plasma sFas-L levels were also negatively correlated with plasma IL-6 levels (Figure 2C). No significant correlation was observed among each separate blood sample and the echocardiographic data in DHCM patients.

Cumulative incidence of CHF in patients with DHCM: Seven DHCM patients were hospitalized for worsening of CHF during the follow-up period (after a mean of 22 months; range, 8-34 months). On the other hand, no HNCM patients experienced any cardiac events for three years. DHCM patients with high E/A (Figure 3A) and sFas levels (Figure 3B) had significantly higher cumulative incidences of $\mathrm{CHF}$ than those of the other populations.

\section{DISCUSSION}

Plasma sFas-L levels in human CHF have been reported to increase or remain unchanged..$^{911)}$ Contrary to these findings, the present study has revealed that plasma sFas-L levels in patients from both HCM groups decreased significantly compared to normal subjects, and the decrease was more marked in DHCM patients. SFas-L is a $26-\mathrm{kDa}$ glycoprotein consisting of the extracellular region of the Fas-L, which is expressed not only in activated T lymphocytes and natural killer cells but also in several organs. ${ }^{21,22)}$ Expression of Fas-L has been reported to be induced during an immune-enhanced response. ${ }^{23)}$ However, the constitutive expression of Fas-L has also been linked to tissue-specific regulation of various immune processes. In this way, the expression of functional Fas-L by some tissues contributes to their immune-privileged status by impairing survival of infiltrating leukocytes. ${ }^{24)}$ Blanco-Colio, et al reported that patients with familial combined hyperlipidemia and carotid atherosclerosis had decreased plasma sFas-L levels, probably due to downregulation of Fas-L expression in endothelial cells, and that mononuclear cell invasion of vessel walls would contribute to the progression of plaque formation. ${ }^{22)}$ In the present study, the plasma levels of sIL$2 \mathrm{R}$, a marker of T-cell activation, did not change in any group. Thus, there is a high probability that the decrease in plasma sFas-L levels in both types of HCM patients in this study might be associated with the downregulation of constitutive Fas-L expression in some tissues. For example, downregulation of constitutive Fas-L expression in vascular endothelial cells facilitates inflammatory cell extravasation and local cell mediated-destruction of cardiac myocytes would tend to take place through the Fas/Fas-L system. In fact, it was previously reported that apoptotic cardiac myocytes were identified with mononuclear cell infiltra- 
tion at the border between the fibrotic and myofibril area of an autopsy heart specimen from a DHCM patient. ${ }^{8)}$

TNF- $\alpha$ or IL-6, which belong to the proinflammatory cytokines family, can be expressed in the myocardium under various forms of stress and are capable of inducing left ventricular hypertrophy, cardiomyopathy and apoptosis in cardiac myocytes. $^{25-31)}$ Furthermore, TNF- $\alpha$ is thought to be intimately involved in the pathogenesis of HCM. ${ }^{32,33)}$ In the present study, plasma TNF- $\alpha$ and IL-6 levels increased in many HNCM patients and the increases were marked in DHCM patients. In addition, the increases in these cytokines tended to deteriorate left ventricular systolic function or LAD in HNCM patients. Thus, these proinflammatory cytokines would have significant detrimental effects on HCM patients, like they do in other cardiac diseases. We also revealed in this study that HNCM patients with low sFas-L levels showed apparent increases in these proinflammatory cytokine levels and that these cytokines tended to increase in proportion to each other. TNF- $\alpha$ is thought to be capable of downregulating the constitutive expression of Fas-L on vascular endothelial cells and regulating leukocyte extravasation. ${ }^{34)}$ Thus, TNF- $\alpha$, which has mainly originated from myocardium, ${ }^{35)}$ might play a critical role in the progression of HCM not only with respect to causing detrimental effects in cardiac function along with IL-6, but also for inducing vascular endothelial dysfunction and facilitating inflammatory cell extravasation by suppressing the constitutive expression of Fas-L.

The Fas antigen is expressed in various tissues, including myocardium, and its soluble form, sFas, is lacking the transmembrane domain. ${ }^{36)}$ The binding of Fas-L, sFas-L, or agonistic anti-Fas antibodies to Fas induces apoptosis to the targeted cell. ${ }^{36-38)}$ Plasma sFas levels with both groups of HCM patients increased significantly compared to the normal subjects in this study. Furthermore, DHCM patients with high sFas levels showed the deterioration of DT, an index of left ventricular diastolic function, and a high incidence of worsening CHF. Thus, plasma sFas levels also increase like in other cardiac diseases in HCM patients and can therefore be used as a prognostic predictor in DHCM patients. However, it is thought plasma sFas levels do not increase significantly in mild cases of CHF, such as those in this study. ${ }^{9,10)}$ Therefore, we believe that the increase in plasma sFas levels in HCM patients would mainly reflect the overexpression of Fas antigen in the myocardium because the influences of tissue hypoxia in other organs, which are a potential source of sFas, due to CHF would be relatively slight in the present patients compared to those in previous reports. Cardiac myocytes overexpressing Fas would increasingly die using an apoptotic system of Fas/Fas-L and myocyte death could be enhanced by mononuclear cell infiltration facilitated by the suppression of constitutive Fas-L expression in vascular endothelial cells. 
The results of the present study indicate that even asymptomatic HNCM patients often demonstrate increases in plasma sFas, TNF- $\alpha$ and IL-6 levels, and that plasma sFas-L levels decreased rather than increased. In addition, in DHCM patients, these findings were more remarkable in spite of their mild CHF state. Although many cases of HCM were suggested to have a genetic cause, ${ }^{39)}$ we were unable to clarify with certainly every phenotype or clinical course from its pathological heterogeneity in HCM patients. Cardiac myocyte destruction and the detrimental effects on the heart caused by the Fas/Fas-L system or proinflammatory cytokines may contribute to the complex features of pathogenesis in HCM.

Study limitations: The sources of these apoptosis mediators as well as the proinflammatory cytokines in human HCM could not be precisely identified in this study because blood samples were only obtained from peripheral veins. Blood samples should be obtained from the coronary sinus, aorta, renal vein, pulmonary artery, and elsewhere and peripheral blood mononuclear cell production of cytokines determined in order to elucidate the sources of these apoptosis mediators and proinflammatory cytokines. Also, the expressions of Fas, Fas-L, TNF- $\alpha$, or IL-6 should be estimated directly from myocardial specimens to validate our hypothesis.

HCM is a very slow progressive disease and basically has a good prognosis. Future longitudinal studies with longer follow-up periods that measure serial apoptosis mediators and proinflammatory cytokines may provide valuable information with respect to identifying cases in transition to the dilated phase or predicting a prognosis in HCM patients.

Conclusions: Slow ongoing cardiac myocyte degeneration due to apoptosis and detrimental effects on cardiac function caused by interactions of the Fas/Fas-L system and proinflammatory cytokines may play an important role in the status of $\mathrm{HCM}$ and its progression to the dilated phase.

\section{REFERENCES}

1. Spirito P, Maron BJ, Bonow RO, Epstein SE. Occurrence and significance of progressive left ventricular wall thinning and relative cavity dilatation in hypertrophic cardiomyopathy. Am J Cardiol 1987; 60: 123-9.

2. Maron BJ. Hypertrophic cardiomyopathy. Curr Probl Cardiol 1993; 18: 639-704. (Review)

3. Emoto R, Yokota Y, Miki T, et al. Prognosis of hypertrophic cardiomyopathy: echocardiographic and postmortem histopathologic study of 30 patients. J Cardiol 1988; 18: 695-703. (Japanese)

4. Maron BJ, Wolfson JK, Epstein SE, Roberts WC. Intramural ("small vessel") coronary artery disease in hypertrophic cardiomyopathy. J Am Coll Cardiol 1986; 8: 545-57.

5. Fananapazir L, Epstein ND. Genotype-phenotype correlations in hypertrophic cardiomyopathy. Insights provided by comparisons of kindreds with distinct and identical beta-myosin heavy chain gene mutations. Circulation 1994; 89: 22-32.

6. Nagata S, Park YD, Minamikawa T, et al. Thallium perfusion and cardiac enzyme abnormalities in patients with familial hypertrophic cardiomyopathy. Am Heart J 1985; 109: 1317-22. 
7. Nishimura T, Nagata S, Uehara T, Hayashida K, Mitani I, Kumita S. Assessment of myocardial damage in dilated-phase hypertrophic cardiomyopathy by using indium-111-antimyosin Fab myocardial scintigraphy. J Nucl Med 1991; 32: 1333-7.

8. Ino T, Nishimoto K, Okubo M, et al. Apoptosis as a possible cause of wall thinning in end-stage hypertrophic cardiomyopathy. Am J Cardiol 1997; 79: 1137-41.

9. Nishigaki K, Minatoguchi S, Seishima M, et al. Plasma Fas ligand, an inducer of apoptosis, and plasma soluble Fas, an inhibitor of apoptosis, in patients with chronic congestive heart failure. J Am Coll Cardiol 1997; 29: 1214-20.

10. Toyozaki T, Hiroe M, Saito T, et al. Levels of soluble Fas in patients with myocarditis, heart failure of unknown origin, and in healthy volunteers. Am J Cardiol 1998; 81: 798-800.

11. Yamaguchi S, Yamaoka M, Okuyama M, et al. Elevated circulating levels and cardiac secretion of soluble Fas ligand in patients with congestive heart failure. Am J Cardiol 1999; 83: 1500-3.

12. Shimizu M, Fukuo K, Nagata S, et al. Increase plasma levels of the soluble form of Fas ligand in patients with acute myocardial infarction and unstable angina pectoris. J Am Coll Cardiol 2002; 39: 585-90.

13. Mann DL, Young JB. Basic mechanisms in congestive heart failure. Recognizing the role of proinflammatory cytokines. Chest 1994; 105: 897-904.

14. Setsuta K, Seino Y, Ogawa T, Ohtsuka T, Seimiya K, Takano T. Ongoing myocardial damage in chronic heart failure is related to activated tumor necrosis factor and Fas/Fas ligand system. Circ J 2004; 68: 747-50.

15. Cheng J, Zhou T, Liu C, et al. Protection from Fas-mediated apoptosis by a soluble form of the Fas molecule. Science 1994; 263: 1759-62.

16. Tanaka M, Suda T, Haze K, et al. Fas ligand in human serum. Nat Med 1996; 2: 317-22.

17. Tsutamoto T, Wada A, et al. Relationship between plasma levels of cardiac natriuretic peptides and soluble Fas: Plasma soluble Fas as a prognostic predictor in patients with congestive heart failure. J Card Fail 2001; 7: 3228.

18. Sasayama S, Matsumori A, Kihara Y. New insights into the pathophysiological role for cytokines in heart failure. Cardiovasc Res 1999; 42: 557-64. (Review)

19. Narula J, Haider N, Virmani R, et al. Apoptosis in myocytes in end-stage heart failure. N Engl J Med 1996; 335: 1182-9.

20. Kang PM, Yue P, Izumo S. New insights into the role of apoptosis in cardiovascular disease. Circ J 2002; 66: 19. (Review)

21. French LE, Hahne M, Virad I, et al. Fas and Fas ligand in embryos and adult mice: ligand expression in several immune-privileged tissues and coexpression in adult tissues characterized by apoptotic cell turnover. J Cell Biol 1996; 133: 335-43.

22. Blanco-Colio LM, Martin-Ventura JL, Sol JM, Diaz C, Hernandez G, Egido J. Decreased circulating Fas ligand in patients with familial combined hyperlipidemia or carotid atherosclerosis: normalization by atorvastatin. $\mathrm{J}$ Am Coll Cardiol 2004; 43: 1188-94.

23. Toyozaki T, Hiroe M, Tanaka M, Nagata S, Ohwada H, Marumo F. Levels of soluble Fas ligand in myocarditis. Am J Cardiol 1998; 82: 246-8.

24. Griffith TS, Brunner T, Fletcher SM, Green DR, Ferguson TA. Fas ligand-induced apoptosis as a mechanism of immune privilege. Science 1995; 270: 1189-92.

25. Bryant D, Becker L, Richardson J, et al. Cardiac failure in transgenic mice with myocardial expression of tumor necrosis factor- $\alpha$. Circulation 1998; 97: 1375-81.

26. Bozkurt B, Kribbs SB, Clubb FJ Jr, et al. Pathophysiologically relevant concentrations of tumor necrosis factor- $\alpha$ promote progressive left ventricular dysfunction and remodeling in rats. Circulation 1998; 97: 1382-91.

27. Krown KA, Page MT, Nguyen C, et al. Tumor necrosis factor alpha-induced apoptosis in cardiac myocyte. Involvement of the sphingolipid signaling cascade in cardiac cell death. J Clin Invest 1996; 98: 2854-65.

28. Hirota H, Yoshida K, Kishimoto T, Taga T. Continuous activation of gp130, a signal-transducing receptor component for interleukin 6-related cytokines, causes myocardial hypertrophy in mice. Proc Natl Acad Sci USA 1995; 92: 4862-6.

29. Birks EJ, Latif N, Owen V, et al. Quantitative myocardial cytokine expression and activation of the apoptotic pathway in patients who require left ventricular assist devices. Circulation 2001; 104: 233-40.

30. Raymond RJ, Dehmer GJ, Theoharides TC, Deliargyris EN. Elevated interleukin-6 levels in patients with asymptomatic left ventricular systolic dysfunction. Am Heart J 2001; 141: 435-8. 
31. Gwechenberger M, Mendoza LH, Youker KA, et al. Cardiac myocytes produce interleukin-6 in culture and in viable border zone of reperfused infarctions. Circulation 1999; 99: 546-51.

32. Matsumori A, Yamada T, Suzuki H, Matoba Y, Sasayama S. Increased circulating cytokines in patients with myocarditis and cardiomyopathy. Br Heart J 1994; 72: 561-6.

33. Nagueh SF, Stetson SJ, Lakkis NM, et al. Decreased expression of tumor necrosis factor- $\alpha$ and regression of hypertrophy after nonsurgical septal reduction therapy for patients with hypertrophic obstructive cardiomyopathy. Circulation 2001; 103: 1844-50.

34. Sata M, Walsh K. TNF- $\alpha$ regulation of Fas ligand expression on the vascular endothelium modulates leukocyte extravasation. Nat Med 1998; 4: 415-20.

35. Feldman AM, Combes A, Wagner D, et al. The role of tumor necrosis factor in the pathophysiology of heart failure. J Am Coll Cardiol 2000; 35: 537-44. (Review)

36. Watanabe-Fukunaga R, Brannan CI, Itoh N, et al. The cDNA structure, expression, and chromosomal assignment of the mouse Fas antigen. J Immunol 1992; 148: 1274-9.

37. Suda T, Takahashi T, Golstein P, Nagata S. Molecular cloning and expression of the Fas ligand, a novel member of the tumor necrosis factor family. Cell 1993; 75: 1169-78.

38. Suda T, Okazaki T, Naito Y, et al. Expression of the Fas ligand in cells of T cell lineage. J Immunol 1995; 154: 3806-13.

39. Marian AJ, Roberts R. The molecular genetic basis for hypertrophic cardiomyopathy. J Mol Cell Cardiol 2001; 33: 655-70. (Review) 